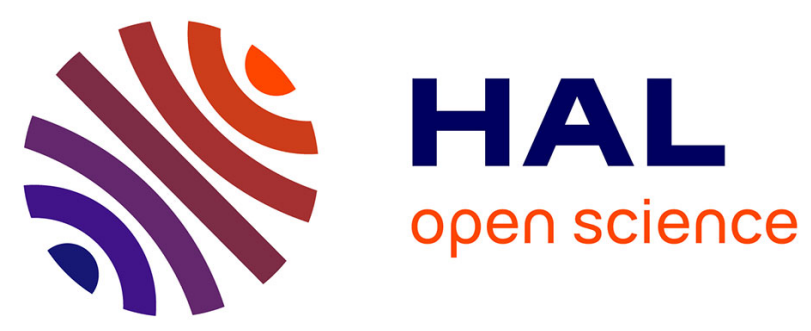

\title{
An attempt to perform inter-band calibration validation for Sentinel-2 using snowy sites such as DomeC in Antarctica
}

\author{
Françoise Viallefont-Robinet
}

\section{- To cite this version:}

Françoise Viallefont-Robinet. An attempt to perform inter-band calibration validation for Sentinel-2 using snowy sites such as DomeC in Antarctica. SPIE Remote Sensing 2019, Sep 2019, Strasbourg, France. 10.1117/12.2532851 . hal-02409754

\section{HAL Id: hal-02409754 https://hal.science/hal-02409754}

Submitted on 13 Dec 2019

HAL is a multi-disciplinary open access archive for the deposit and dissemination of scientific research documents, whether they are published or not. The documents may come from teaching and research institutions in France or abroad, or from public or private research centers.
L'archive ouverte pluridisciplinaire HAL, est destinée au dépôt et à la diffusion de documents scientifiques de niveau recherche, publiés ou non, émanant des établissements d'enseignement et de recherche français ou étrangers, des laboratoires publics ou privés. 


\title{
An attempt to perform inter-band calibration validation for Sentinel 2 using snowy sites such as Dome $\mathrm{C}$ in Antarctica
}

\author{
F. Viallefont-Robinet* \\ ONERA, Université de Toulouse F-31055 Toulouse - France
}

\begin{abstract}
In the frame of the monitoring of Sentinel 2 MSI sensors in orbit, a method for inter-band calibration validation, using Antarctic site, has been developed and tested. The method relies on the directional hemispheric reflectance found in ASTER data base. In order to consider that the spectral shape of the directional directional reflectance is the same as the directional hemispheric one, wavelengths have to be limited to the spectral range below $0.9 \mu \mathrm{m}$. Thus this method cannot perform the inter-band calibration for the whole set of spectral bands of MSI but only from B1 to B8A. Results for B9 seem also correct although the central wavelength is a little bit beyond $0.9 \mu \mathrm{m}$.
\end{abstract}

Given a clear day acquisition on DomeC site, a first set of computations of Top Of Atmosphere (TOA) reflectances for MSI spectral bands is performed using the three ASTER reflectances corresponding respectively to small, medium and coarse grain size for snow. The spectral reflectances are normalized by the B2 value. The comparison of the three normalized TOA reflectances to the actual one given by MSI enables to select the right snow grain size. Then the second step is the atmospheric parameters adjustment. Water content is assessed thanks to B9 band centered at $0.945 \mu \mathrm{m}$ and ozone is assessed thanks to mainly B3 band centered at $0.56 \mu \mathrm{m}$. For a clear day, the aerosol optical thickness is weak and thus the TOA reflectance is not very sensitive to the more or less low abundance of continental aerosols. The ratio of normalized reflectance given by MSI to the normalized reflectance given by the computation is assessed for each spectral band between B1 and B9 and is compared to the inter-band calibration validations performed with other methods.

Keywords: Inter-band, Calibration, Validation, remote sensing, Sentinel 2, Antarctica

*francoise.viallefont@onera.fr; phone +33 562252610

\section{INTRODUCTION}

Calibration and validation (Cal/Val) is performed regularly all the sensor life long to monitor, optimize and control the image quality. Inter-band calibration validation is part of this Cal/Val activity and is particularly important for application requiring a high accuracy on the band ratios. The inter-band validation can be derived from absolute calibration using methods such as Rayleigh scattering over ocean surface [1][2], ground-based reflectance measurements [3][4][5], or Deep Convective Clouds [6]. Most methods cannot perform the validation for the whole set of spectral bands, for the moment, only the method relying on ground based measurements can. Despite a partial coverage of the spectral domain, it is really interesting to assess the image quality through various independent methods. Inter-band validation using Antarctic site such as DomeC is another independent method to assess the band ratio accuracy.

The inter-band validation is performed for the L1C product level delivering Top Of Atmosphere (TOA) reflectance. The requirements are demanding as the ratio between two spectral bands should be equal to the expected value $\pm 3 \%$. This implies to assess this ratio with a very high accuracy. 


\section{DATA OVERVIEW}

\subsection{Sentinel 2 main features}

A description of the Multi Spectral Instrument (MSI) of Sentinel 2 satellite can be found in [7]. Regarding the inter-band calibration validation, the MSI most important features are the relative spectral responses. They can be found at: https://earth.esa.int/web/sentinel/document-library/content/-/article/sentinel-2a-spectral-responses. For each spectral band, Table 1 recalls its central wavelength, the band width and the associated Ground Sampling Distance.

\begin{tabular}{|c|c|c|c|}
\hline Band number & Central wavelength $(\mathrm{nm})$ & Bandwidth $(\mathrm{nm})$ & Ground Sampling Distance $(\mathrm{m})$ \\
\hline 1 & 443 & 20 & 60 \\
\hline 2 & 490 & 65 & 10 \\
\hline 3 & 560 & 35 & 10 \\
\hline 4 & 665 & 30 & 10 \\
\hline 5 & 705 & 15 & 20 \\
\hline 6 & 740 & 15 & 20 \\
\hline 7 & 783 & 20 & 20 \\
\hline 8 & 842 & 115 & 10 \\
\hline 8 A & 865 & 20 & 20 \\
\hline 9 & 945 & 20 & 60 \\
\hline 10 & 1375 & 30 & 60 \\
\hline 11 & 1610 & 90 & 20 \\
\hline 12 & 2190 & 180 & 20 \\
\hline
\end{tabular}

Table 1: MSI spectral bands and associated Ground Sampling Distance

\subsection{Data}

Sentinel2 MPA reports

The mean Top Of Atmosphere (TOA) reflectance over Dome C for each spectral band is provided by the Mission Performance Center along with the solar zenith and azimuth angles and the viewing zenith and azimuth angles. As data need to be cloud-free, only a few DomeC acquisitions are processed. The Table 1 summarizes the Antarctic data involved in this study.

\begin{tabular}{|c|c|c|}
\hline Date & Time & relative orbit \\
\hline $07 / 10 / 2016$ & $23 \mathrm{~h} 48 \mathrm{~m} 02 \mathrm{~s}$ & 44 \\
\hline $20 / 01 / 2018$ & $00 \mathrm{~h} 17 \mathrm{~m} 51 \mathrm{~s}$ & 30 \\
\hline $20 / 01 / 2018$ & $23 \mathrm{~h} 47 \mathrm{~m} 51 \mathrm{~s}$ & 44 \\
\hline $27 / 01 / 2018$ & $00 \mathrm{~h} 05 \mathrm{~h} 01 \mathrm{~s}$ & 130 \\
\hline $27 / 01 / 2018$ & $23 \mathrm{~h} 38 \mathrm{~m} 81 \mathrm{~s}$ & 1 \\
\hline $30 / 01 / 2018$ & $00 \mathrm{~h} 17 \mathrm{~m} 51 \mathrm{~s}$ & 30 \\
\hline
\end{tabular}

Table 2: Summary of the Sentinel 2 A Antarctic data used in the study

\section{Snow reflectance}

The method described in section 3 requires as input the spectral reflectance of the ground for the area acquired by the sensor. For snow, three spectral directional-hemispheric reflectances can be found in the ASTER spectral library https://speclib.jpl.nasa.gov/library corresponding respectively to small, medium and coarse grain size of snow. These reflectances are plotted in Figure 1. The information given with the spectra mention that the 
reflectances are obtained thanks to a model for the wavelengths between 0.3 and $2.08 \mu \mathrm{m}$ and thanks to measurements between 2.08 and $14 \mu \mathrm{m}$.

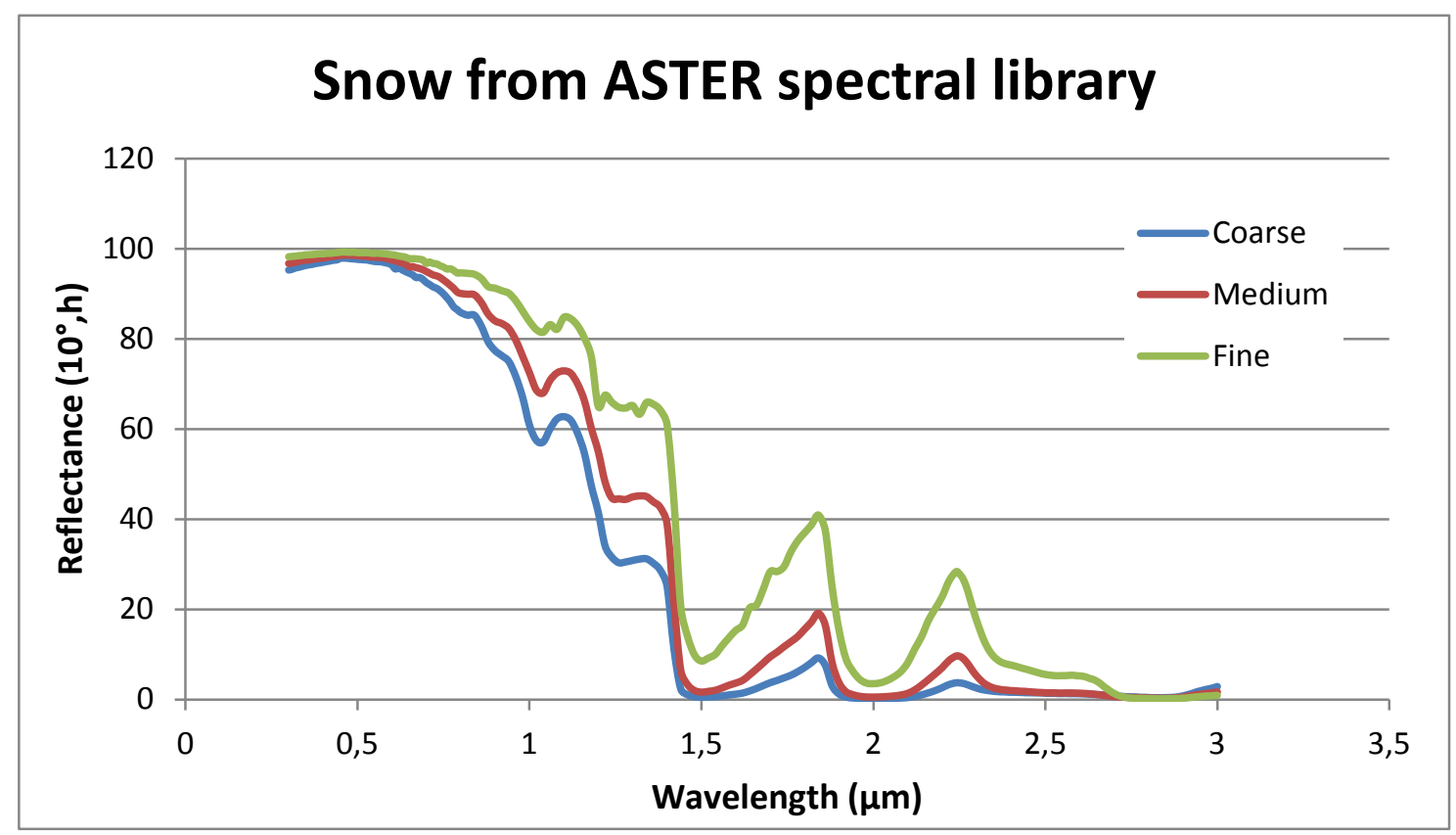

Figure 1: Directional-hemispheric reflectance of snow for three grain sizes, from the ASTER spectral library

\section{METHOD}

\subsection{Directional-directional reflectance}

A preliminary work for this method is to derive the directional-directional Bottom Of Atmosphere (BOA) from the BOA directional-hemispheric reflectance found in the ASTER library. Hudson et al. paper [8] presents the reflectance factor, RF, normalized by the albedo, nRF. This normalized reflectance factor appears to be fairly independent from the wavelength for the high albedo values that is to say for wavelengths between 0.35 and 0.9 $\mu \mathrm{m}$.

Thus, for a wavelength $\lambda \leq 0.9 \mu \mathrm{m}$, it comes:

$$
\rho^{d d}\left(\lambda, \theta_{s}, \theta_{v}, \varphi_{s}, \varphi_{v}\right) \approx \alpha(\lambda) \times n R F\left(\theta_{s}, \theta_{v}, \varphi_{s}, \varphi_{v}\right)
$$

$\rho^{\mathrm{dd}}$ being the directional directional reflectance,

$\theta \mathrm{s}$ and $\theta \mathrm{v}$ being respectively the solar and the viewing zenith angles,

$\varphi s$ and $\varphi v$ being the solar and the viewing azimuth angles,

$\alpha$ being the albedo.

The directional hemispheric reflectance can then be expressed as:

$$
\rho^{d h}\left(\lambda, \theta_{s}, \varphi_{s}\right)=\iint \rho^{d d}\left(\lambda, \theta_{s}, \theta_{v}, \varphi_{s}, \varphi_{v}\right) d \theta_{v} d \varphi_{v} \approx \alpha(\lambda) \iint n R F\left(\theta_{s}, \theta_{v}, \varphi_{s}, \varphi_{v}\right) d \theta_{v} d \varphi_{v}
$$

This implies that the spectral shape is driven by the albedo and it is the same shape for $\rho^{\mathrm{dd}}$ and $\rho^{\mathrm{dh}}$. Thus, it is possible to express the directional-directional reflectance as:

$$
\rho^{d d}\left(\lambda, \theta_{s}, \theta_{v}, \varphi_{s}, \varphi_{v}\right) \approx c\left(\theta_{s}, \theta_{v}, \varphi_{s}, \varphi_{v}\right) \times \rho^{d h}\left(\lambda, \theta_{s}, \theta_{v}, \varphi_{s}\right)
$$

$c\left(\theta_{s}, \theta_{v}, \varphi_{s}, \varphi_{v}\right)$ being a coefficient,

This can be rewritten in a convenient way as:

$$
\rho^{d d}\left(\lambda, \theta_{s}, \theta_{v}, \varphi_{s}, \varphi_{v}\right) \approx R C\left(\theta_{s}, \theta_{v}, \varphi_{s}, \varphi_{v}\right) \times \rho^{d h}\left(\lambda, \theta_{s}=10^{\circ}, \varphi_{s}\right)
$$


$R C\left(\theta_{s}, \theta_{v}, \varphi_{s}, \varphi_{v}\right)$ being a Reflectance Coefficient,

$\rho^{d h}\left(\lambda, \theta_{s}=10^{\circ}, \varphi_{s}\right)$ being the reflectance found in the ASTER spectral library.

This means that the spectral shape is not impacted by the angular configuration, only the level is. Thus, the directional - directional reflectance spectral shape is the same as the directional - hemispheric reflectance spectral shape.

\subsection{Grain size and reflectance coefficient}

A classical way to calibrate or to validate calibration is to perform vicarious calibration. It consists in comparing TOA reflectance coming from the sensor to TOA reflectance computed thanks to BOA reflectance measurements along with atmospheric characterization. This way to proceed can be adapted to inter-band calibration validation using BOA reflectance of snow. As no measurement can be done as the satellite overpasses, the reflectance is chosen among the existing ones. The issue is to choose between fine, medium and coarse grain size.

For the date of acquisition, thanks to snow directional - hemispheric reflectance ASTER model for 3 grain sizes, a computation of the TOA reflectance is done using 6S and a priori values for the atmospheric parameters. The computed TOA reflectance is then compared to the TOA reflectance found in the MPA report. This leads to the curves of Figure 2. In order to identify more easily the grain size corresponding to the S2A image, the TOA reflectance ratio (band $\mathrm{Bi} /$ band B2) is computed for each i and plotted Figure 3 . This enables to select the medium grain size as the one of the snow observed on 07/10/2016.

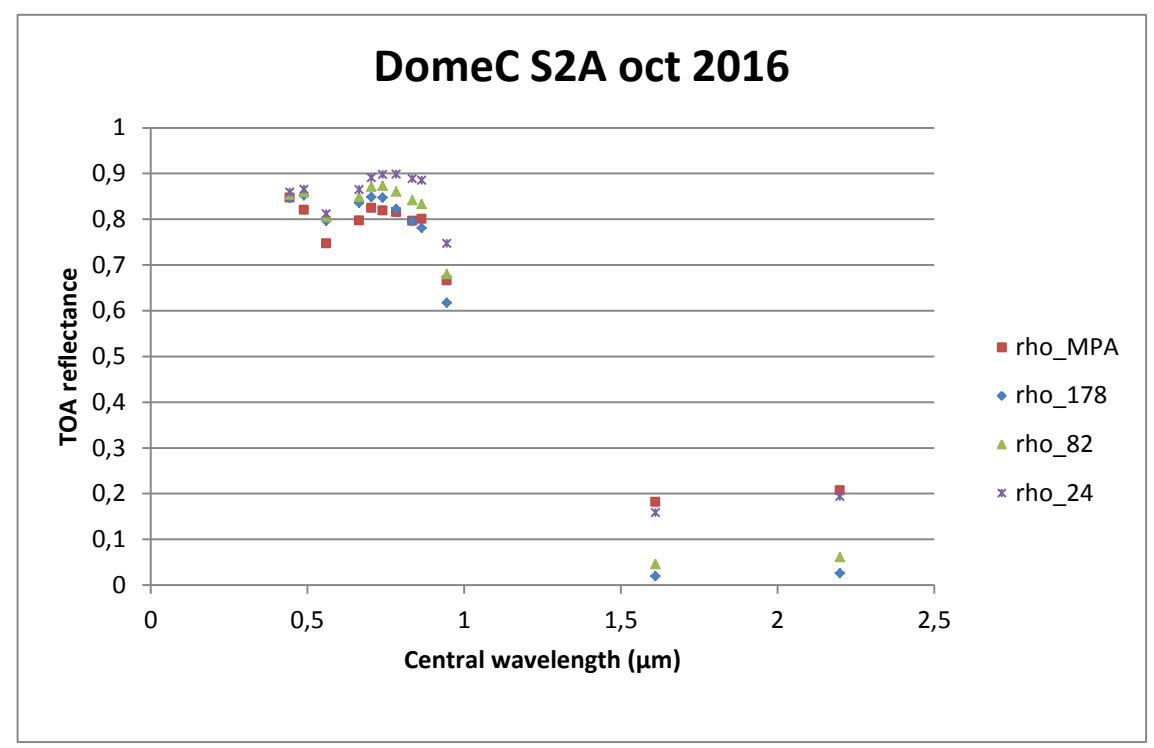

Figure 2 : Comparison of the computed and observed TOA reflectances 


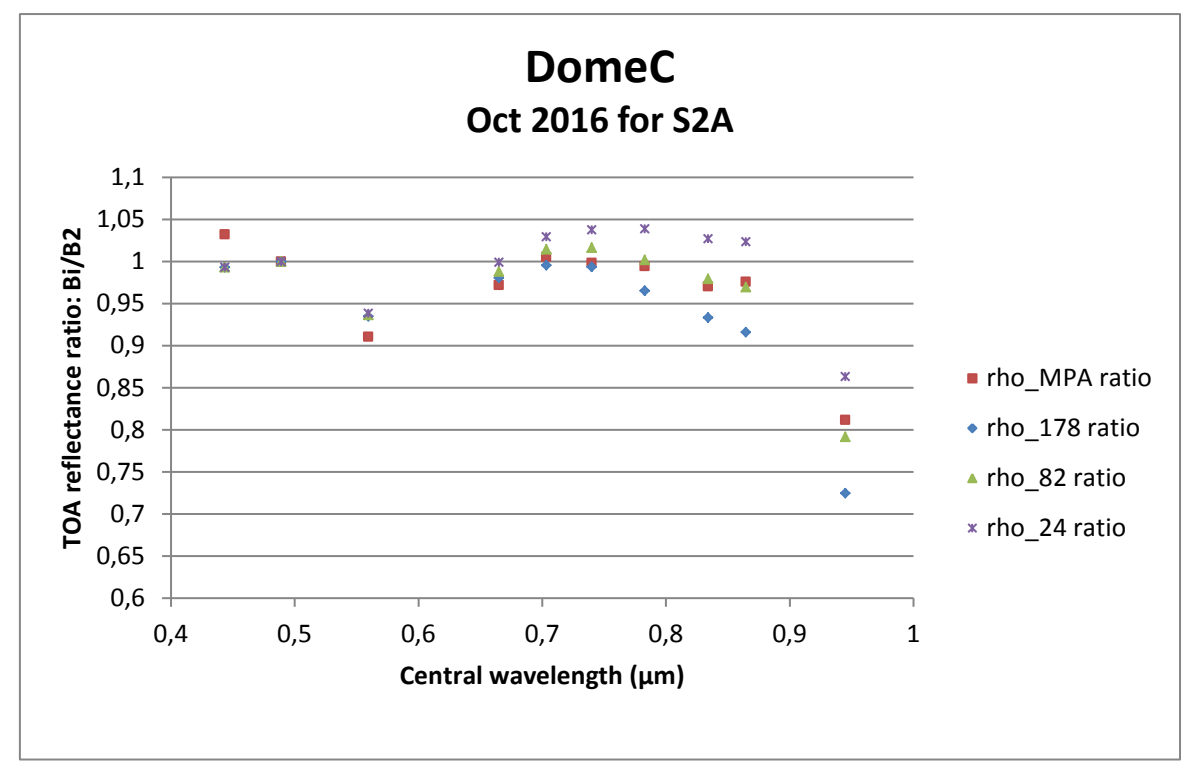

Figure 3: Comparison of the computed and observed reflectance ratios $\mathrm{Bi} / \mathrm{B2}$

The reflectance coefficient is then assessed by choosing the reflectance coefficient (among 0.85, 0.90, 0.95 and 1.00) which put the MPA report reflectance and the $6 \mathrm{~S}$ reflectance in agreement mainly for B2 and B4 as shown Figure 4. As this factor is close to 1, despite the atmosphere effect, it doesn't impact the band ratios as shown Figure 5.

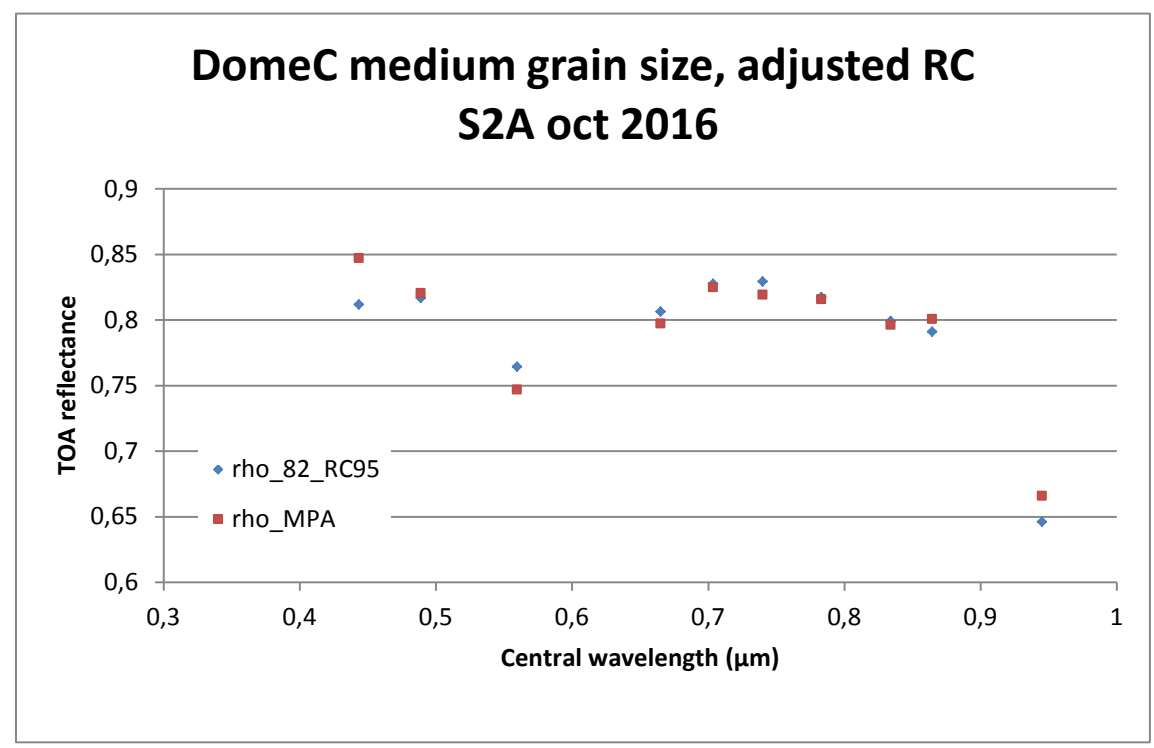

Figure 4: Comparison of computed and observed TOA reflectance for the chosen RC 


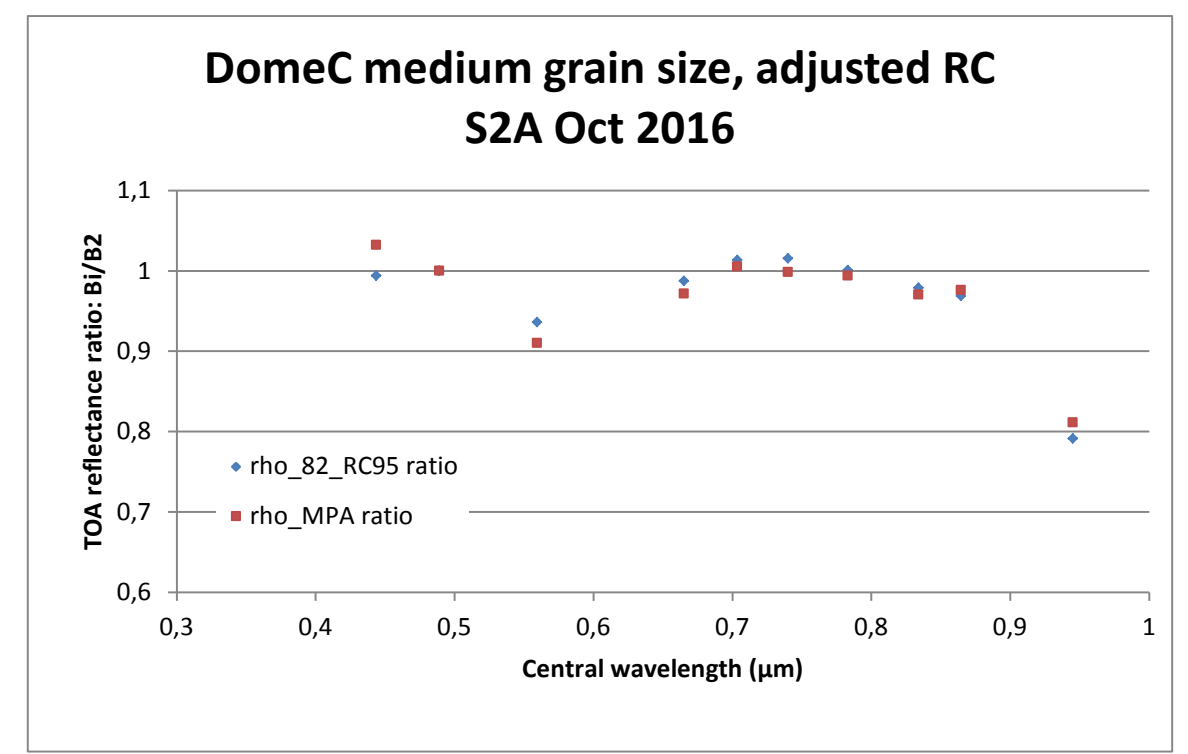

Figure 5: Comparison of computed and observed TOA reflectance ratios $\mathrm{Bi} / \mathrm{B} 2$ for the chosen $\mathrm{RC}$

\subsection{Ozone and water vapor}

As there is no atmospheric characterization available at the acquisition date and time, the atmospheric parameters have to be found. Complementary computations, for the selected grain size and RC, are performed for 25 and 26 values respectively for water vapor and ozone.

The water vapor content can be adjusted with (and for) B9, as shown by the comparison of Figure 6 and Figure 4.

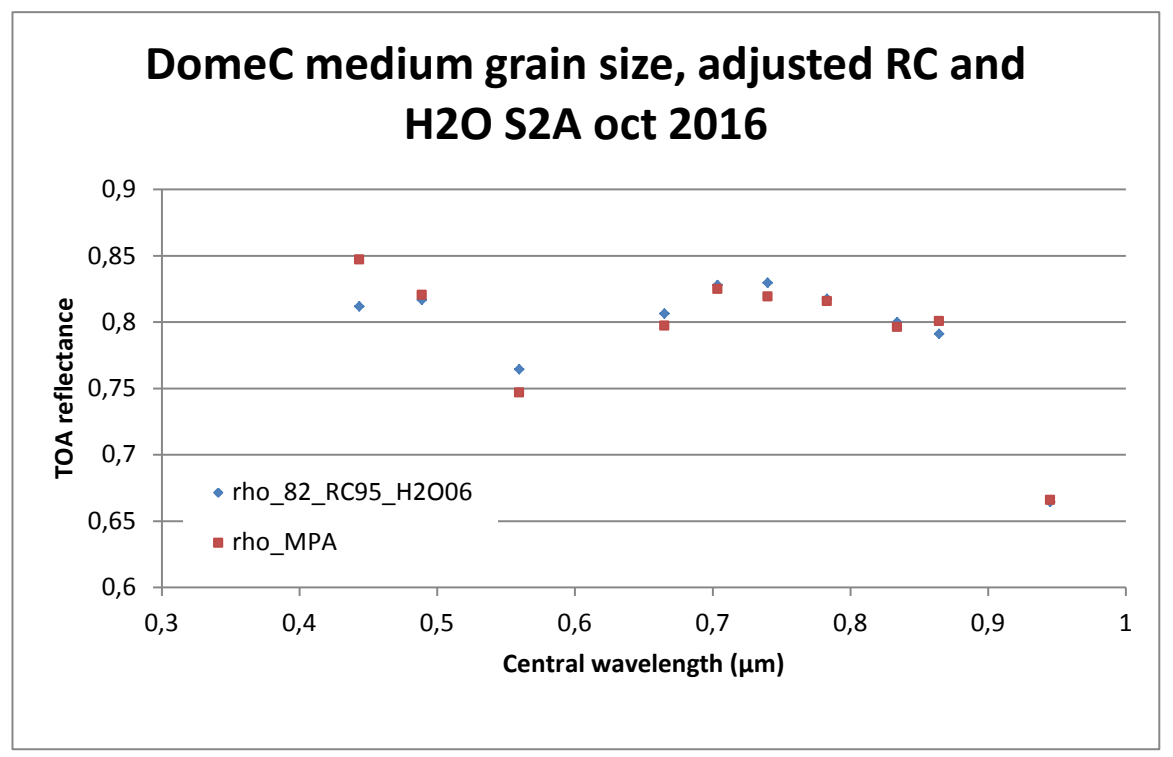

Figure 6: Comparison of computed and observed TOA reflectance ratios Bi/B2 for the chosen water vapor content

The adjustment of the ozone content can be done looking at B2, B3 and B4. The corresponding reflectances are presented Figure 7. 


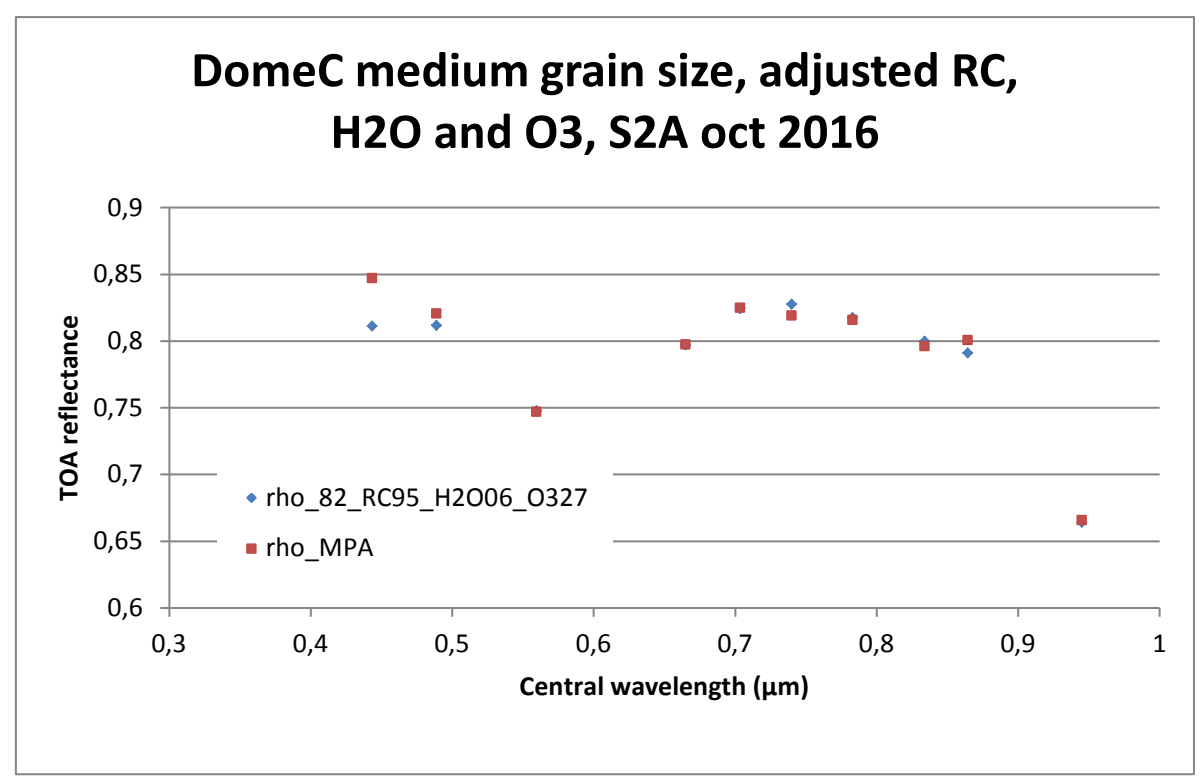

Figure 7: Comparison of computed and observed TOA reflectance ratios $\mathrm{Bi} / \mathrm{B} 2$ for the chosen water vapor and ozone contents

\section{RESULTS}

The inter-band calibration validation result for B2 as reference band is presented Figure 8. This first result is in agreement, except for B1, with results presented in [1] Figure 28 (a) and (b).

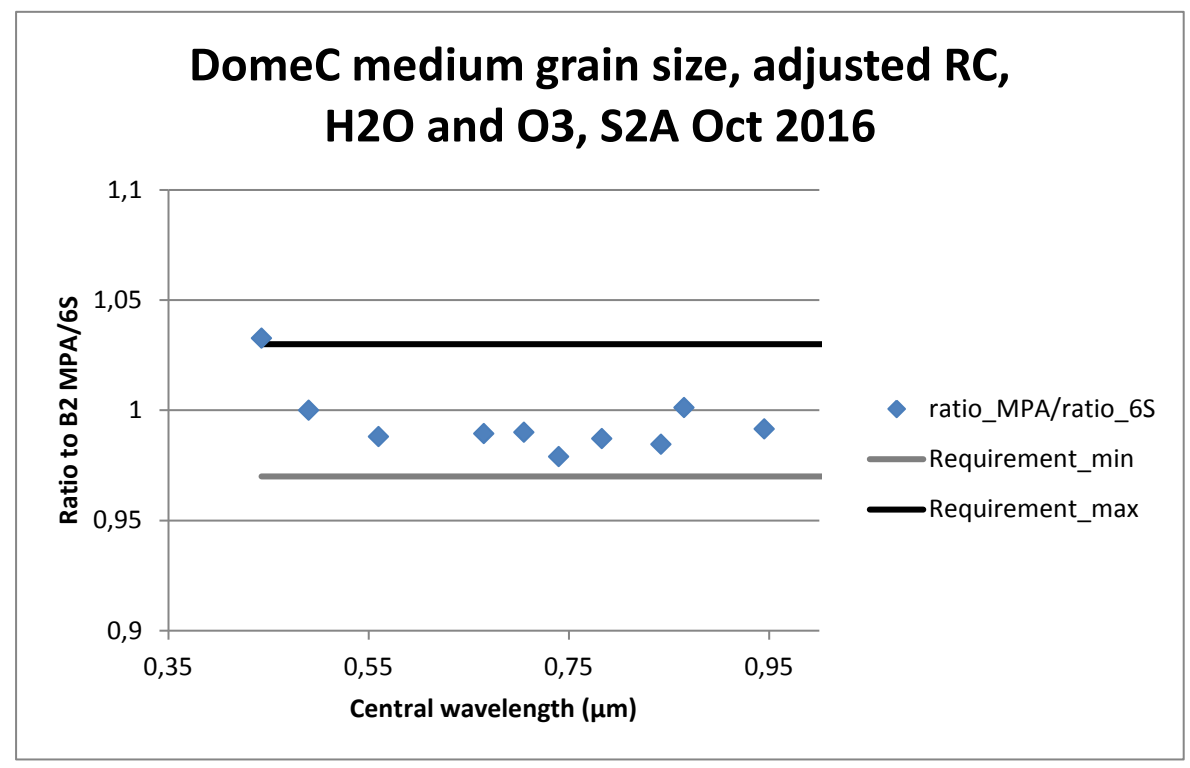

Figure 8: Comparison of computed (6S) and observed (MPA) band ratios (Bi/B2) : MPA/6S

The mean result for the data of January 2018 is given Figure 9. The bar corresponds to the standard deviation over the 5 acquisitions processed and gives the precision of the method. The unexpected behavior of B1 is still there, even worse. It seems that the ratio $\mathrm{Bi} / \mathrm{B} 2$ for $\mathrm{I} \geq 4$ has been shifted upward by 0.01 to 0.02 . But the global shape remains in agreement with the previous one. 


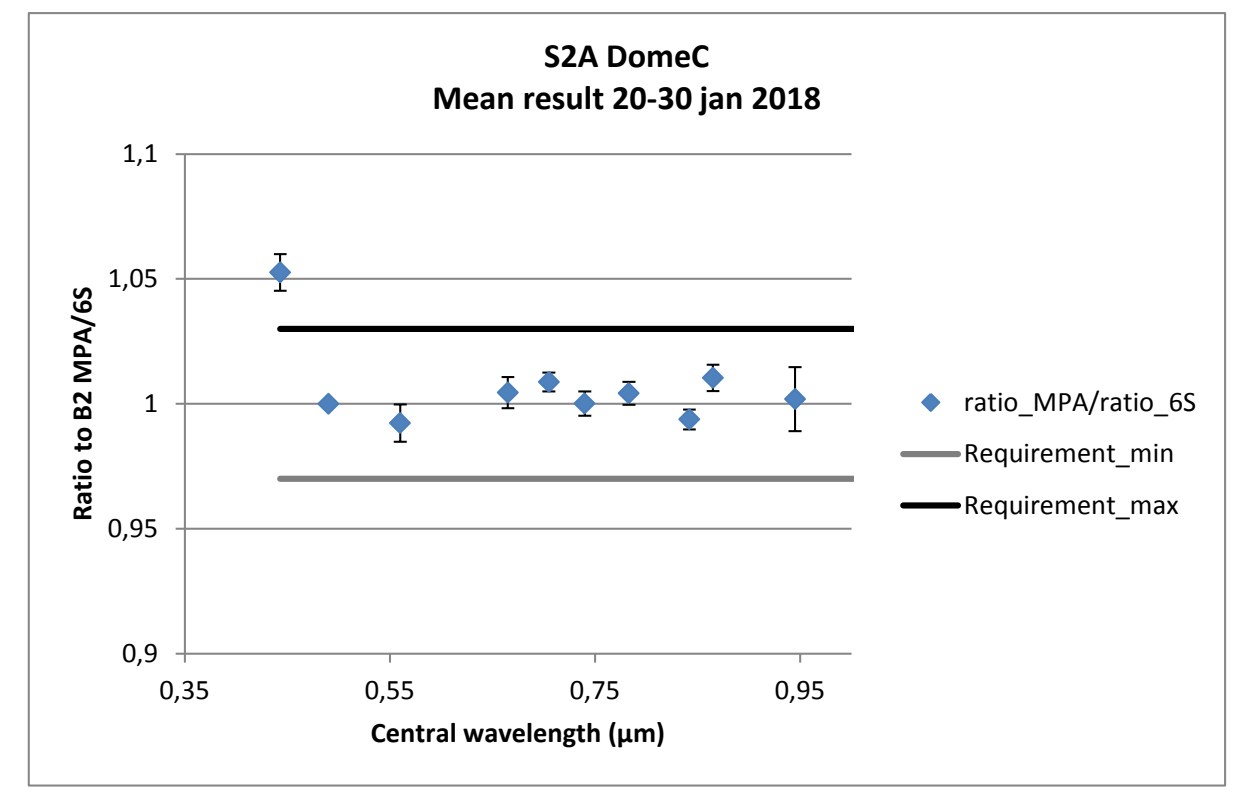

Figure 9: Comparison of computed (6S) and observed (MPA) band ratios (Bi/B2) for January 2018

\section{CONCLUSION}

A new method has been proposed and tested for inter-band validation between 0.35 and $0.9 \mu \mathrm{m}$. It seems to be valid until $0.95 \mu \mathrm{m}$. The results obtained for S2 are in agreement with those obtained with other methods except for B1. In the B1 case, the unexpected result cannot be explained by atmospheric parameters. It might be due to a difference between the actual spectrum and the model. This difference could come from the state of the snow or from the model.

\section{ACKNOWLEDGMENTS}

This work was carried out in the framework of the Sentinel 2 CalVal activity under the lead of CS. I wish to acknowledge Laetitia Pessiot (CS) as Sentinel-2 MPC Service manager, Olivier Thépaut (formerly at CS) and then Catherine Bouzinac (CS), Sentinel-2 MPC Cal/Val Coordinator, for their help particularly for computer science, Benjamin Francesconi (Thales Alenia Space) and then Marion Neveu Van Malle (Thales Alenia Space) as coordinator for L1 product validation, Ferran Gascon (ESA) as ESA Sentinel-2 Mission Manager and Valentina Boccia (ESA) as Sentinel-2 Data Quality and Cal/Val Manager and all the Cal/Val team. I also wish to warmly acknowledge the MPCC team which produces the MPA reports for Cal/Val.

\section{REFERENCES}

[1] Gordon, H.R.; Wang, M. Retrieval of Water-Leaving Radiance and Aerosol Optical Thickness over the Oceans with SeaWiFS: A Preliminary Algorithm. Appl. Opt., 33, 443-452 (1994).

[2] Vermote, E.; Santer, R.; Deschamps, P.Y.; Herman, M. In-flight Calibration of Large Field-of-View Sensors at Short Wavelengths using Rayleigh Scattering. Int. J. Remote Sens., 13, 3409-3429 (1992).

[3] Slater, P.N.; Biggar, S.F.; Holm, R.G.; Jackson, R.D.; Mao, Y.; Moran, M.S.; Palmer, J.M.; Yuan, B. Reflectance-and radiance-based methods for the in-flight absolute calibration of multispectral sensors. Remote Sens.Environ., 22, 11-37 (1987).

[4] Thome, K.J.; Helder, D.L.; Aaron, D.; Dewald, J.D. Landsat-5 TM and Landsat-7 ETM+ absolute radiometric calibration using the reflectance-based method. IEEE Trans. Geosci. Remote Sens., 42, 2777-2785 (2004). 
[5] Czapla-Myers, J.; McCorkel, J.; Anderson, N.; Thome, K.; Biggar, S.; Helder, D.; Aaron, D.; Leigh, L.;Mishra, N. The Ground-Based Absolute Radiometric Calibration of Landsat 8. Remote Sens., 7, 600-626 (2015)

[6] Fougnie, B.; Bach, R. Monitoring of Radiometric Sensitivity Changes of Space Sensors Using Deep Convective Clouds: Operational Application to PARASOL. IEEE Trans. Geosci. Remote Sens., 47, 851-861 (2009).

[7] Gascon F., Bouzinac C., Thépaut O., Jung M., Francesconi B., Louis J., Lonjou V., Lafrance B. , Massera S., Gaudel-Vacaresse A., Languille F., Alhammoud B., Viallefont F., Pflug B., Bieniarz J., Clerc S., Pessiot L., Trémas T., Cadau E., De Bonis R., Isola C., Martimort P. and Fernandez V., “Copernicus Sentinel-2A Calibration and Products Validation Status," Remote Sens. 2017, 9, 584; doi:10.3390/rs9060584..

[8] Hudson S. R., Warren S. G., Brandt R. E., Grenfell T. C., Six D, “Spectral bidirectional reflectance of Antarctic snow: measurements and parametrization,” J. Geophys. Res., Vol. 11, D18106 (2006). 